\title{
Accessibility Density - A New Trend for Transportation Planning
}

\author{
Eyad Trabulsi ${ }^{1}$, and Akmal Abdelfatah ${ }^{1}$
}

\begin{abstract}
The new trends of development of mega-scale projects determine new challenges in urban planning, transportation and construction engineering. The traditional planning tools must address the new challenges associated with mega-scale projects. One of the significant challenges is accessibility which is defined by Litman[1] as people's ability to reach goods, services and activities. The term "Accessibility Density" (AxeDe) proposed in this paper is a single metric that captures the accessibility level from one entrance to the numerous destinations, activities, and functions that exist within a development, with reference to a certain threshold of units. The theory and application of the proposed AxeDe concept have been examined on Mall of the Emirates (MOE); a mega-scale shopping mall as a case study in the city of Dubai. AxeDe represents a potential shift in the planning process by addressing a transportation challenge relevant to access to/from mega-scale developments, where walking is the common mode of transportation. It aims at knowing how accessibility can be evaluated/ measured within mega-scale pedestrian-dominated developments. The outcomes illustrated the impact of design on accessibility. Further demonstrations of the observed results have been suggested with further research to develop the concept by addressing otherfactors of accessibility and types of land-use.
\end{abstract}

Keywords-Accessibility, Indoor Transportation, Accessibility Density, Transportation Planning.

\section{INTRODUCTION}

$\mathrm{T}$ HE significant scale of new projects, in modern metropolitan cities, has been adding another level of complication on projects and cities' planning. The traditional methods of transportation planning have been recently falling short in addressing the upcoming changes in developments' trends. Those methods are appropriate for small and medium scales of projects. Mega-scale projects represent black boxes within a city map. In order to ensure quality of results in transportation planning and modelling, new tools are required to address the accumulative errors associated with neglecting significant pedestrian's trips that take place internally within mega-scale projects.

Batty [2] hints to the need to reassess the top-down approach, in transportation planning networks, to more appropriate and practical bottom-up approach.This approach suggests determining networks' planning and modelling at a lower scale prior to addressing higher levels of transportation networks.

\footnotetext{
${ }^{1}$ American University of Sharjah, University City, Sharjah, United Arab Emirates
}

AxeDe suggests a potential shift in transportation planning for metropolitan cities where mega-scale projects are developed. It represents an effective measurement tool for planners to control lower levels of detail while working on higher scales of cities' planning.

Accessibility Density (AxeDe) is a proposed single metric that quantitatively captures the accessibility level (density) from one entrance to the numerous destinations, activities, and functions that exist within a pedestrian-dominated development, with reference to a certain threshold of units (e.g. time).

AxeDe is to be captured per each project's entrance as it represents the interim point between external and internal transportation. AxeDe is measured by dividing the sum of accessible areas from each entrance of a project, within a proposed fixed threshold (e.g. 150 seconds), over the total destination areaswithin a project.

Fig. 1 presents a schematic illustration of how to measure AxeDe for one of the project's entrance.

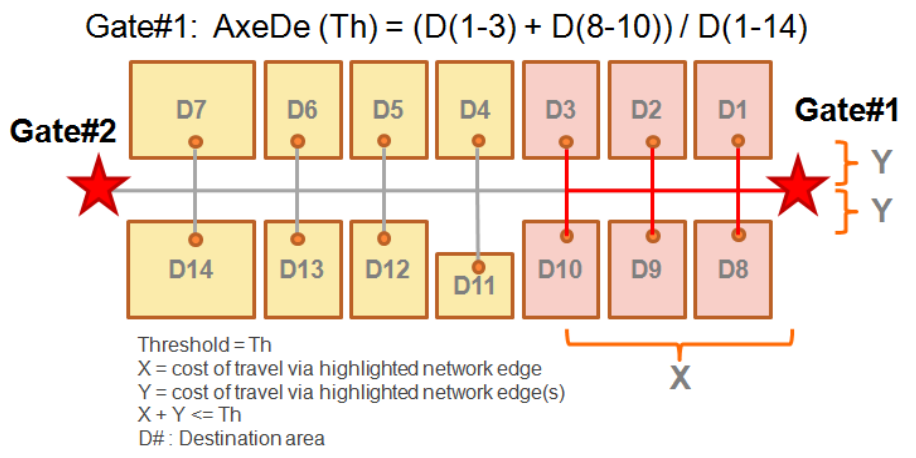

Fig. 1: Measuring AxeDe

\section{PRoBlem StATEMENT}

Mega-scale projects impose new challenges on transportation planning and modelling. An increasinglysignificant part of trips started being non-properly addressed while conducting transportation modelling; such error used to be reasonable to neglect in small to medium scales of projects.

Fig. 2 illustrates the non-addressed part of trips (indoor trip) where an origin or a destination exists within a mega-scale project. 


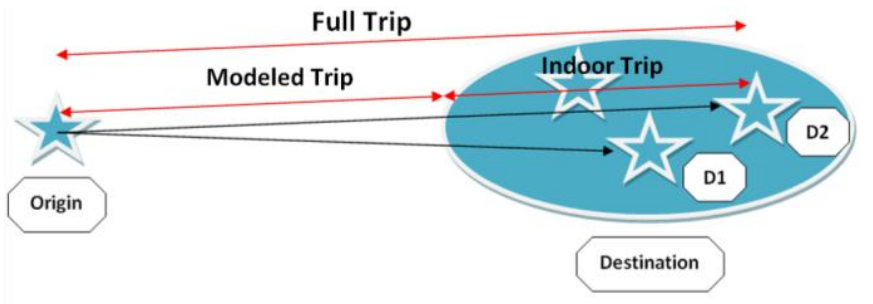

Fig. 2: Modelled trip vs. non-addressed indoor trip within a megascale project

It is imperative to indicate to the characteristics of indoor trips, which differ from external trips. Indoor trips take place within 3 dimensional networks, which contain different transportation means (i.e. walking, lifts, escalators, etc).

\section{The VAlue OF AXEDE}

With a new approach towards modern cities' transportation planning and modeling, AxeDe represents a potential valuable futuristic planning tool that supports transportation planners in addressing low-level transportation challenges at a higher-level of transportation planning and modelling. Therefore, AxeDe is foreseen to highly benefit:

- Regulators/Authorities - via the introduction of new regulations and measures to enforce more accessible projects and higher harmonization between infrastructure supply and projects' demands/attraction.

- Property Developers - via the introduction of higher standards at pre-design phase. Developers benefit by achieving better functionality and, consequently, more attractive projects via design innovation.

- End users - via easier access and, consequently, less stressful transportation.

To achieve its benefits, AxeDe is channeled via two major industry lines:

- Transportation planning.

- Architectural design.

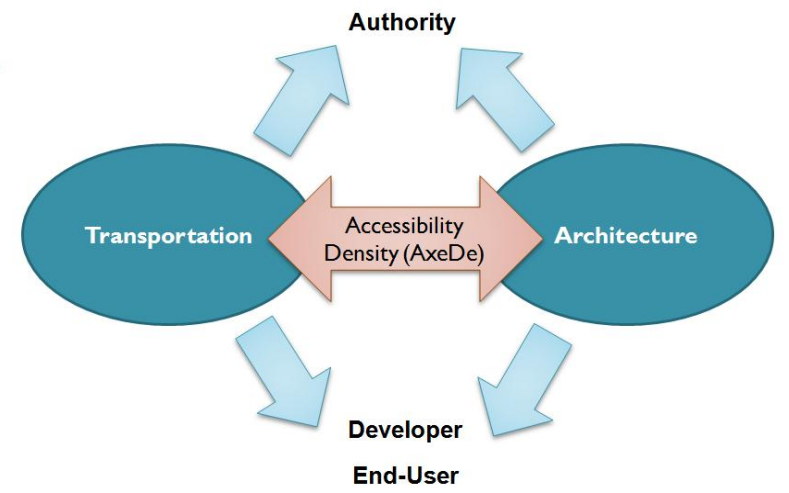

Fig. 3: AxeDe Value towards authorities, developers and end-users.

Fig. 3 shows who might benefit from AxeDe and how benefits are channeled via their relevant industry lines and businesses.

\section{LITERATURE REVIEW}

Taking into consideration that AxeDe is a new concept for which very limited literature has been published earlier; however, AxeDe is based on mature sciences where a valuable literature exists in the fields of design, accessibility, pedestrians' standards, as well as mapping and routing.

The literature review for AxeDe is essential in building its model. Many standards and parameters, required for AxeDe model, have been extracted from relevant researches, as follows:

- The first application of AxeDe adopts average value(s) of pedestrians' attributes (i.e. walking speed in shopping centres - average of $1 \mathrm{~m} / \mathrm{sec}$ ) as what was identified in global literature. Local parameters are recommended to be identified and considered in later applications as depicted in Fig. 4. [3]

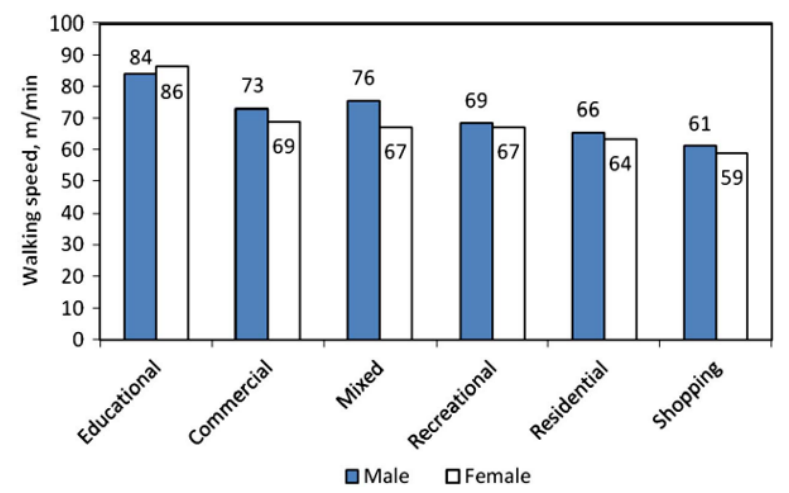

Fig. 4: Effect of Land Use and Gender.[3]

- As concluded by Neutens[4], regardless the Floating Catchment Area (FCA) which does not represent real geometric distance, travel time is the preferred measure of accessibility metrics as it is more capable of better addressing free walking and travel via vertical transportation means (i.e., escalators, travellators) among different levels, as shown inFig. 5.

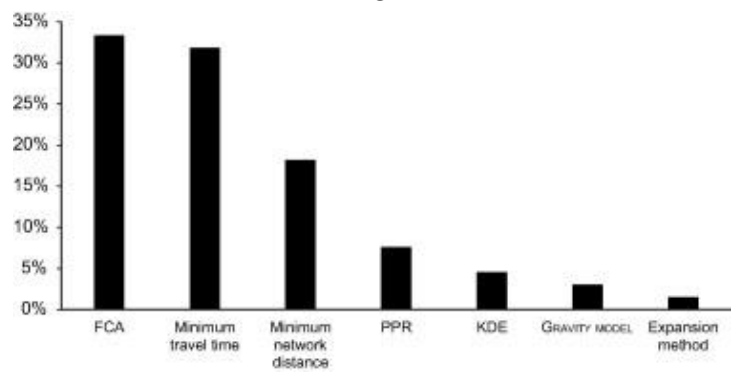

Fig. 5: Frequency in the Use of Accessibility Metrics.[4]

- Worboys[5], Khan and Kolbe [6], Pradhan [7] and Brummit and Shafer [8], suggested the development of a hybrid model combining topological and geometric features. This will be represented via graph-based network consisting of nodes and segments (taken from Pre-processed CAD drawings to Shape Files) and has to be linked to the logical model of buildings and to external network transfer points (i.e. parking lots, public transportation stations/stops, etc).

- Level of Detail (LOD) of the model is to consider LOD2, as indicated by Hagedorn et al. [9], via highlighting only zones which are useful for the application. The different levels of detail are presented in Fig. 6. 

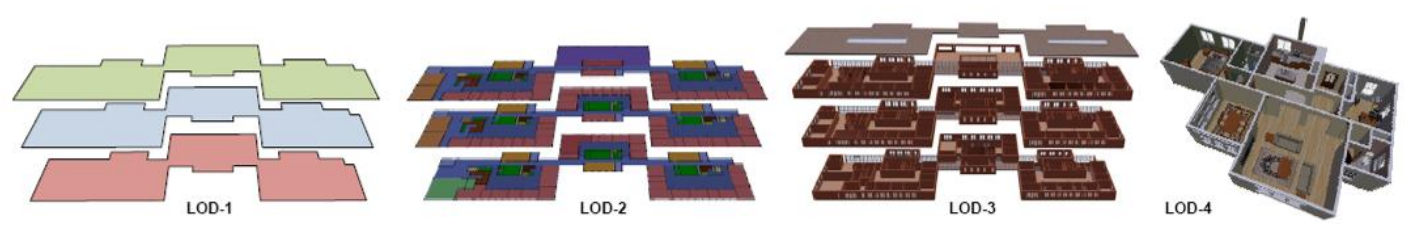

Fig. 6: Indoor model instances at different LODs.[9]

- The Weighted Indoor Routing Graph (WIRG) method is used in AxeDe application, as proposed by Goetz and Zipf [9], by considering the following:

$\circ$ Centerline in the corridors/pathways.

- Point-of-Interest/obstacle nodes in the main halls/rooms, with consideration to the model proposed by Yao et al. [10]

- One-way / two-way paths.

- Vertical building parts (i.e. escalators, elevators ... etc.).

WIRG is a graph model that comprises topologic, semantic and geometricfeatures.

As inferred by Lorenz et al. [11], a 3-D indoor space could be represented via 2-D maps/layouts. The third dimension is to be reflected via vertical transportation means' connectivity (i.e. escalators and elevators as major means of indoor transportation in shopping malls) which represents gateways (containing quantitative and qualitative attributes) connecting different levels.

\section{METHODOLOGY}

Fig. 7 illustrates a procedural flowchart for the proposed AxeDe methodology. On one side, AxeDe standards and parameters are required to stay up-to-date via on-going literature review that benefits the AxeDe concept. On the other side, the project's layouts and the proposed technology (that impacts travel cost) are to be identified, then pre-processed, via $C A D$ as well as spatial software(s), in order to build AxeDe skeleton (network) to run the model. It is imperative to conduct the following tasks throughout the 2 levels of preprocessing:

- CAD pre-processing:

- Subdivide main indoor space.

- Add start points at entrances.

- Add connecting Spaces Nodes and classify them by color/layer.

- Add end points at destinations.

- Draw Dual/Path using the centerline approach.

- Classify transfer edges.

- Spatial/GIS pre-processing:

- Tag Nodes and Edges with special attributes to compute cost-of-travel.

- Prepare Network to be routable.
- Test AxeDe concept for each pedestrian's gate using different thresholds of units (i.e. 120 seconds, 300 seconds).

In order to conclude AxeDe values, thresholds are used to sum up all areas that are accessible from each entrance (via an Origin Destination matrix), within an assigned cap of cost of travel (threshold). The resulted sum (for each entrance, per threshold) is to be divided over the total destinations' areas of the project, which shall indicate the AxeDe value (for entrance) within specific threshold. Example: for Gate01,AxeDe (120s) = XX \%.

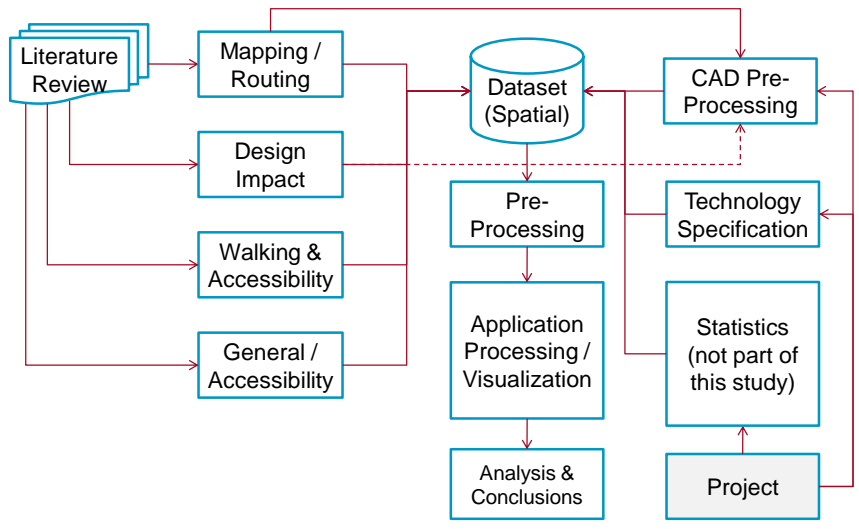

Fig. 7: Proposed AxeDe Framework's Procedural Flowchart.

\section{AXEDE MODEl APPLICATION's DEVELOPMENT AND RESULTS}

The AxeDe first application has been conducted on a major shopping mall in Dubai, called; Mall of the Emirates (MoE). In this paper, an illustration of AxeDe impact on maps is visualized only for entrances of one level of the shopping center. However, the AxeDe values are presented and analyzed for all entrances/levels.

Applying AxeDe model on MoE's ground level layout, from the variant gates and within an assigned threshold of $120 \mathrm{~s}$, results access coverage maps. For MoE's ground level gates, two samplesof resulted maps are illustrated. Fig. 8 shows the accessible units within the ground floor from the gates in the ground floor for a threshold of 120 seconds. Also, the access from the gates in ground level to stores in the first level (L1) for the same threshold (120 seconds) is presented in Fig. 9. 


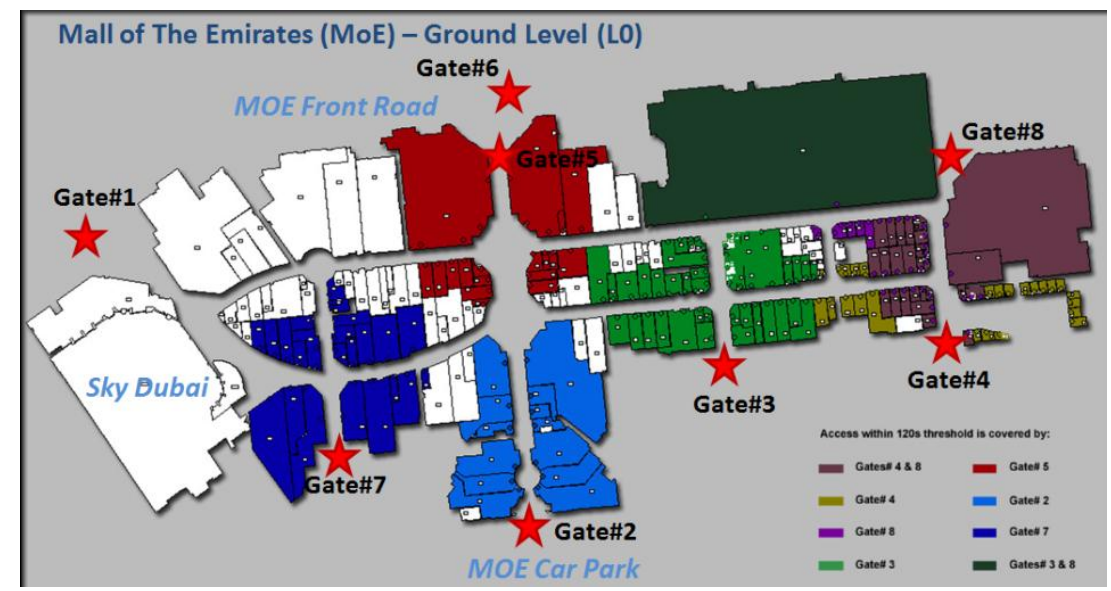

Fig. 8: Accessible Units/Coverage of L0Gates within a Threshold of 120 seconds (L0).

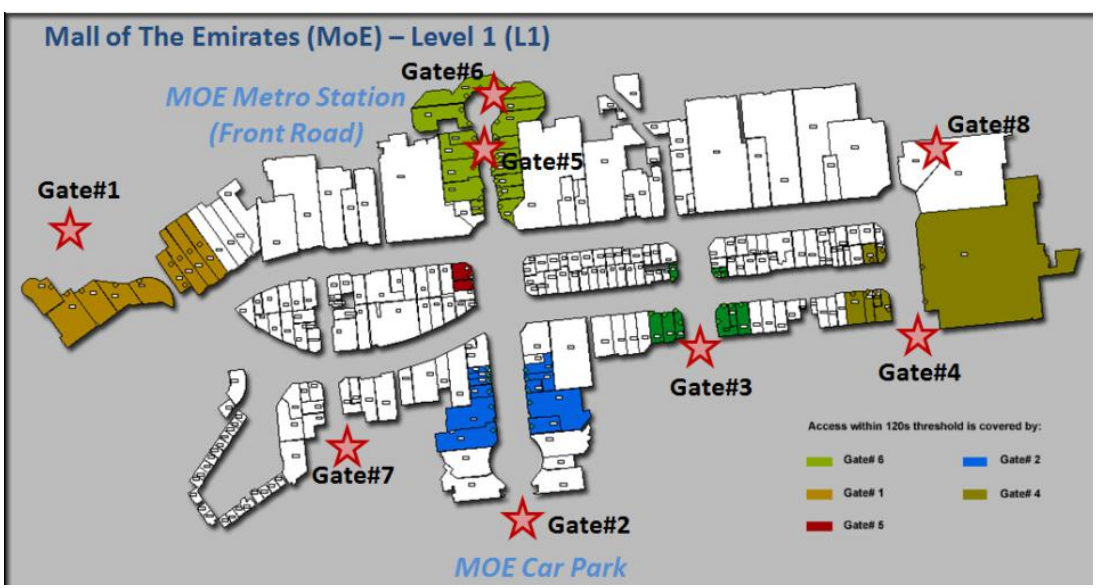

Fig. 9: Accessible Units/Coverage of L0Gates within a Threshold of 120 seconds (L1).

Table 1 illustrates AxeDe values, for all MoE gates, within two tentative thresholds (i.e. 120s and 300s):

TABLE I: AXEDE RATIOS OF MOE GATES PER LEVEL (L0, L1 AND L2) - CLASSIFIED PER THRESHOLD (120S AND 300S)

\begin{tabular}{|c|c|c|c|c|c|c|c|c|c|c|c|c|c|c|c|c|c|}
\hline Gates & $0 \mathrm{~g} 01$ & L0g02 & $\mathrm{LOg} 03$ & $\mathrm{LOg} 04$ & L0g05 & L0g06 & og07 & L0g08 & L1g09 & L1g10 & L1g11 & $\mathrm{L} 1 \mathrm{~g} 12$ & $\operatorname{L1g} 13$ & $\mathrm{~L} 2 \mathrm{~g} 14$ & $\mathrm{~L} 2 \mathrm{~g} 15$ & $\mathrm{~L} 2 \mathrm{~g} 16$ & L2g17 \\
\hline $\begin{array}{l}120 \mathrm{~s} \\
\text { Threshold } \\
\text { AxeDe (120s) }\end{array}$ & $2.0 \%$ & $5.7 \%$ & $\begin{array}{c}11.8 \\
\%\end{array}$ & $9.7 \%$ & $4.9 \%$ & $2 \%$ & $4.0 \%$ & $12.6 \%$ & $2.7 \%$ & $3.7 \%$ & $\begin{array}{c}13.3 \\
\%\end{array}$ & $12.8 \%$ & $1.8 \%$ & $2.6 \%$ & $1.5 \%$ & $5.8 \%$ & $5.8 \%$ \\
\hline $\begin{array}{l}300 \mathrm{~s} \\
\text { Threshold } \\
\text { AxeDe (300s) }\end{array}$ & $29.2 \%$ & $58.1 \%$ & $\begin{array}{c}67.3 \\
\%\end{array}$ & $43.8 \%$ & $74.4 \%$ & $42.9 \%$ & $56.9 \%$ & $43.8 \%$ & $58.5 \%$ & $\begin{array}{c}53.8 \\
\%\end{array}$ & $\begin{array}{c}74.8 \\
\%\end{array}$ & $44.8 \%$ & $\begin{array}{c}41.3 \\
\%\end{array}$ & $8.7 \%$ & $\begin{array}{c}64.6 \\
\%\end{array}$ & $\begin{array}{c}38.7 \\
\%\end{array}$ & $\begin{array}{c}55.0 \\
\%\end{array}$ \\
\hline
\end{tabular}

As shown in Table 1, it is obvious that accessibility is influenced by the common features of entrances, such as:

- Entrances with high AxeDe values (highly connected), within low thresholds (i.e., 120s), have common features, such as:

$\circ \quad$ Very close to junction(s)

- Close to major shops (mega stores with large floor area)

- Entrances with high AxeDe values (highly connected), within high thresholds (i.e., 300s), have common features, such as:

$\bigcirc$ Centrally located in the project

- Not far from major junctions but not conditionally very close

- Well connected to other levels

\section{CONCLUSION}

AxeDe represents a proposed planning tool to quantitatively-measure the level of accessibility within megascale developments. AxeDe benefits from multiple fields of research (i.e., mapping and routing, pedestrians' accessibility, walkability, design impact on accessibility, etc) in order to enhance the pre-planning process of mega-scale projects and/or to improve accessibility within planned/existing projects. It may support planners to ensure appropriate level of accessibility within internal networks, via simple figures, without a need to look into the details of a project. This also may lead to achieve better harmonization between external and internal transportation networks, at planning phase; it may minimize bottlenecks along these networks, and consequently 
save on costs (associated with expanding non-adequately planned infrastructures), and to ensure smooth traffic flow along the different segments of transportation networks (i.e. external, parking, internal).

The proposed framework of AxeDe is recommended to be further tested to be introduced as a robust tool, commissioned by authorities/developers, to develop adequately-planned projects and infrastructures.

\section{REFERENCES}

[1] T. Litman, "Evaluating Accessibility for Transportation Planning Measuring People's Ability To Reach Desired Goods and Activities," Retrieved March 12, 2016, from http://www.vtpi.org/access.pdf

[2] M. Batty, "Network Geography: Relations, Interactions, Scaling and Spatial Processes in GIS," Working Papers Series (Paper 63), p. 23., 2003.

[3] R. Rastogi, I. Thaniarasu, S. Chandra, "Design Implications of Walking Speed for Pedestrian Facilities," Journal of Transportation Engineering, 137(10), 687-696, 2011. http://dx.doi.org/10.1061/(ASCE)TE.1943-5436.0000251

[4] T. Neutens, "Accessibility, equity and health care: review and research directions for transport geographers, " Journal of Transport Geography, 43, 14-27, 2015. http://dx.doi.org/10.1016/j.jtrangeo.2014.12.006

[5] M. Worboys, "Modeling Indoor Space," Proceedings of the $3^{\text {rd }}$ ACM SIGSPATIAL International Workshop on Indoor Spatial Awareness, 16,2011 http://dx.doi.org/10.1145/2077357.2077358

[6] A. Khan, T. Kolbe, "Subspacing based on connected opening spaces and for different locomotion types using geometric and graph based representation in multilayered space-event model (MLSEM)," ISPRS Annals of the Photogrammetry, Remote Sensing and Spatial Information Sciences, 173-185, 2013.

[7] S. Pradhan, "Semantic location," Personal Technologies, 4 (4), 213 216, 2000. http://dx.doi.org/10.1007/BF02391560

[8] B. Brumitt, S. Shafer, "Topological world modeling using semantic spaces," Location Modeling for Ubiquitous Computing, 55-61, 2001.

[9] B. Hagedorn, M. Trapp, T. Glander, \& J. Dollner, "Towards an Indoor Level-of-Detail Model for Route Visualization," Tenth International Conference on Mobile Data Management: Systems, Services and Middleware, 692-697, 2009. http://dx.doi.org/10.1109/MDM.2009.118

[10] M. Goetz, M., A. Zipf, "Formal Definition of a User-Adaptive and Length-Optimal Routing Graph for Complex Indoor Environments," Geo-spatial Information Science, 14 (2), 119-128, 2011. http://dx.doi.org/10.1007/s11806-011-0474-3

[11] L. Yao, L. Sun, W. Wang, H. Xiong, "Concept Layout Model of Transportation Terminals," Discrete Dynamics in Nature and Society, 2012, 1-8, 2012 http://dx.doi.org/10.1155/2012/304868

[12] B. Lorenz, B., H. J"urgenOhlbach, E. Stoffel, "A Hybrid Spatial Model for Representing Indoor Environments," Web and Wireless Geographical Information Systems: 6th International Symposium, W2GIS 2006, pp. 102-112, 2006. 